\title{
A Study of Nonlinear Fractional Differential Equations of Arbitrary Order with Riemann-Liouville Type Multistrip Boundary Conditions
}

\author{
Bashir Ahmad, ${ }^{1}$ Sotiris K. Ntouyas, ${ }^{2}$ and Ahmed Alsaedi ${ }^{1}$ \\ ${ }^{1}$ Department of Mathematics, Faculty of Science, King Abdulaziz University, P.O. Box 80203, Jeddah 21589, Saudi Arabia \\ ${ }^{2}$ Department of Mathematics, University of Ioannina, 45110 Ioannina, Greece
}

Correspondence should be addressed to Bashir Ahmad; bashirahmad_qau@yahoo.com

Received 28 November 2012; Accepted 19 January 2013

Academic Editor: José Tenreiro Machado

Copyright (C) 2013 Bashir Ahmad et al. This is an open access article distributed under the Creative Commons Attribution License, which permits unrestricted use, distribution, and reproduction in any medium, provided the original work is properly cited.

We develop the existence theory for nonlinear fractional differential equations of arbitrary order with Riemann-Liouville type boundary conditions involving nonintersecting finite many strips of arbitrary length. Our results are based on some standard tools of fixed point theory. For the illustration of the results, some examples are also discussed.

\section{Introduction}

The subject of fractional calculus has recently developed into a hot topic for the researchers in view of its numerous applications in the field of physics, mechanics, chemistry, engineering, and so forth. One can find the systematic progress of the topic in the books ([1-6]). A significant characteristic of a fractional-order differential operator distinguishing it from the integer-order differential operator is that it is nonlocal in nature, that is, the future state of a dynamical system or process involving fractional derivative depends on its current state as well its past states. In fact, this feature of fractionalorder operators has contributed towards the popularity of fractional-order models, which are recognized as more realistic and practical than the classical integer-order models. In other words, we can say that the memory and hereditary properties of various materials and processes can be described by differential equations of arbitrary order. There has been a rapid development in the theoretical aspects such as periodicity, asymptotic behavior, and numerical methods for fractional equations. For some recent work on the topic, see ([7-23]) and the references therein. In particular, Ahmad et al. [22] studied nonlinear fractional differential equations and inclusions of arbitrary order with multistrip boundary conditions.

In this paper, we continue the study initiated in [22] and consider a boundary value problem of fractional differential equations of arbitrary order $q \in(n-1, n], n \geq 2$ with finite many multistrip Riemann-Liouville type integral boundary conditions:

$$
\begin{gathered}
{ }^{c} D^{q} x(t)=f(t, x(t)), \quad t \in[0, T], \\
x(0)=0, \quad x^{\prime}(0)=0, \ldots, x^{(n-2)}(0)=0, \\
x(T)=\sum_{i=1}^{m} \gamma_{i}\left[I^{\beta i} x\left(\eta_{i}\right)-I^{\beta i} x\left(\zeta_{i}\right)\right],
\end{gathered}
$$

where ${ }^{c} D^{q}$ denotes the Caputo fractional derivative of order $q$, $f$ is a given continuous function, $I^{\beta_{i}}$ is the Riemann-Liouville fractional integral of order $\beta_{i}>0, i=1,2, \ldots, m, 0<\zeta_{1}<$ $\eta_{1}<\zeta_{2}<\eta_{2}<\ldots<\zeta_{m}<\eta_{m}<T$, and $\gamma_{i} \in \mathbb{R}$ are suitable chosen constants.

Regarding the motivation of the problem, we know that the strip conditions appear in the mathematical modeling of 
certain real world problems, for instance, see [24, 25]. In [22], the authors considered the nonlocal strip conditions of the form:

$$
\begin{array}{r}
x(1)=\sum_{i=1}^{n-2} \alpha_{i} \int_{\zeta_{i}}^{\eta_{i}} x(s) d s, \quad 0<\zeta_{i}<\eta_{i},<1, \\
i=1,2, \ldots,(n-2) .
\end{array}
$$

In the problem (1), we have introduced Riemann-Liouville type multistrip integral boundary conditions which can be interpreted as the controller at the right-end of the interval under consideration is influenced by a discrete distribution of finite many nonintersecting sensors (strips) of arbitrary length expressed in terms of Riemann-Liouville type integral boundary conditions. For some engineering applications of strip conditions, see ([26-32]).

The main objective of the present study is to develop some existence results for the problem (1) by using standard techniques of fixed point theory. The paper is organized as follows. In Section 2 we discuss a linear variant of the problem (1), which plays a key role in developing the main results presented in Section 3. For the illustration of the theory, we have also included some examples.

\section{Preliminary Result}

Let us begin this section with some basic definitions of fractional calculus [2-4].

Definition 1. If $g(t) \in A C^{n}[a, b]$, then the Caputo derivative of fractional order $q$ is defined as

$$
\begin{aligned}
{ }^{c} D_{a^{+}}^{q} g(t) & =\frac{1}{\Gamma(n-q)} \int_{a}^{t}(t-s)^{n-q-1} g^{(n)}(s) d s \\
& =I_{a^{+}}^{n-q} D^{n} g(x), \quad n-1<q<n, n=[q]+1,
\end{aligned}
$$

where $[q]$ denotes the integer part of the real number $q$. For details, see Theorem 2.1 ([4, page 92]). Here $A C^{n}[a, b]$ denote the space of real valued functions $g(t)$ which have continuous derivatives up to order $n-1$ on $[a, b]$ such that $g^{n-1}(t) \in$ $A C[a, b]$.

Definition 2. The Riemann-Liouville fractional integral of order $q$ is defined as

$$
I^{q} g(t)=\frac{1}{\Gamma(q)} \int_{0}^{t} \frac{g(s)}{(t-s)^{1-q}} d s, \quad q>0,
$$

provided the integral exists.

The following result associated with a linear variant of problem (1) plays a pivotal role in establishing the main results.
Lemma 3. For $h \in C[0, T]$, the fractional boundary value problem

$$
\begin{gathered}
{ }^{c} D^{q} x(t)=h(t), \quad t \in[0, T], q \in(n-1, n] \\
x(0)=0, \quad x^{\prime}(0)=0, \ldots, x^{(n-2)}(0)=0, \\
x(T)=\sum_{i=1}^{m} \gamma_{i}\left[I^{\beta i} x\left(\eta_{i}\right)-I^{\beta i} x\left(\zeta_{i}\right)\right],
\end{gathered}
$$

has a unique solution $x(t) \in A C^{n}[0, T]$ given by

$$
\begin{aligned}
& x(t)= \frac{1}{\Gamma(q)} \int_{0}^{t}(t-s)^{q-1} h(s) d s \\
&-\frac{t^{n-1}}{\lambda \Gamma(q)} \int_{0}^{T}(T-s)^{q-1} h(s) d s \\
&+\frac{t^{n-1}}{\lambda \Gamma(q)} \sum_{i=1}^{m} \frac{\gamma_{i}}{\Gamma\left(\beta_{i}\right)} \quad\left[\int_{0}^{\eta_{i}} \int_{0}^{s}\left(\eta_{i}-s\right)^{\beta_{i}-1}\right. \\
& \quad-\int_{0}^{\zeta_{i}} \int_{0}^{s}\left(\zeta_{i}-s\right)^{\beta_{i}-1}(s-u)^{q-1} \\
& \times(s-u)^{q-1} h(u) d u d s \\
&\times h(u) d u d s],
\end{aligned}
$$

where

$$
\lambda=\left(T^{n-1}-\sum_{i=1}^{m} \gamma_{i} \frac{\left(\eta_{i}^{\beta_{i}+n-1}-\zeta_{i}^{\beta_{i}+n-1}\right) \Gamma(n)}{\Gamma\left(\beta_{i}+n\right)}\right) \neq 0 .
$$

Proof. The general solution of fractional differential equations in (5) can be written as

$$
x(t)=\frac{1}{\Gamma(q)} \int_{0}^{t}(t-s)^{q-1} h(s) d s-c_{0}-c_{1} t-\cdots-c_{n-1} t^{n-1} .
$$


Using the given boundary conditions, it is found that $c_{0}=0$, $c_{1}=0, \ldots, c_{n-2}=0$. Applying the Riemann-Liouville integral operator $I^{\beta_{i}}$ on (8), we get

$$
\begin{aligned}
& I^{\beta_{i}} x(t) \\
& =\frac{1}{\Gamma\left(\beta_{i}\right)} \int_{0}^{t}(t-s)^{\beta_{i}-1} \\
& \times\left(\frac{1}{\Gamma(q)} \int_{0}^{s}(s-u)^{q-1} h(u) d u-c_{n-1} s^{n-1}\right) d s \\
& =\frac{1}{\Gamma\left(\beta_{i}\right) \Gamma(q)} \int_{0}^{t} \int_{0}^{s}(t-s)^{\beta_{i}-1}(s-u)^{q-1} h(u) d u d s \\
& -c_{n-1} \frac{1}{\Gamma\left(\beta_{i}\right)} \int_{0}^{t}(t-s)^{\beta_{i}-1} s^{n-1} d s .
\end{aligned}
$$

Using the condition $x(T)=\sum_{i=1}^{m} \gamma_{i}\left[I^{\beta_{i}} x\left(\eta_{i}\right)-I^{\beta_{i}} x\left(\zeta_{i}\right)\right]$, together with the fact that

$$
\frac{1}{\Gamma\left(\beta_{i}\right)} \int_{0}^{t}(t-s)^{\beta_{i}-1} s^{n-1} d s=\frac{t^{\beta_{i}+n-1} \Gamma(n)}{\Gamma\left(\beta_{i}+n\right)},
$$

we obtain

$$
\begin{gathered}
\frac{1}{\Gamma(q)} \int_{0}^{T}(T-s)^{q-1} h(s) d s-c_{n-1} T^{n-1} \\
=\sum_{i=1}^{m} \frac{\gamma_{i}}{\Gamma(q) \Gamma\left(\beta_{i}\right)} \\
\times\left[\int_{0}^{\eta_{i}} \int_{0}^{s}\left(\eta_{i}-s\right)^{\beta_{i}-1}(s-u)^{q-1} h(u) d u d s\right. \\
\left.\quad-\int_{0}^{\zeta_{i}} \int_{0}^{s}\left(\zeta_{i}-s\right)^{\beta_{i}-1}(s-u)^{q-1} h(u) d u d s\right] \\
-c_{n-1} \sum_{i=1}^{m} \gamma_{i} \frac{\left(\eta_{i}^{\beta_{i}+n-1}-\zeta_{i}^{\beta_{i}+n-1}\right) \Gamma(n)}{\Gamma\left(\beta_{i}+n\right)},
\end{gathered}
$$

which yields

$$
\begin{gathered}
c_{n-1}=\frac{1}{\lambda \Gamma(q)} \int_{0}^{T}(T-s)^{q-1} h(s) d s \\
-\frac{1}{\lambda \Gamma(q)} \sum_{i=1}^{m} \frac{\gamma_{i}}{\Gamma\left(\beta_{i}\right)} \\
\times\left[\int_{0}^{\eta_{i}} \int_{0}^{s}\left(\eta_{i}-s\right)^{\beta_{i}-1}\right. \\
\quad \times(s-u)^{q-1} h(u) d u d s \\
\quad \int_{0}^{\zeta_{i}} \int_{0}^{s}\left(\zeta_{i}-s\right)^{\beta_{i}-1}(s-u)^{q-1} \\
\times h(u) d u d s],
\end{gathered}
$$

where $\lambda$ is given by (7). Substituting the values of $c_{0}$, $c_{1}, \ldots, c_{n-2}, c_{n-1}$ in (8), we obtain (6). This completes the proof.

\section{Main Results}

Let $\mathscr{C}:=C([0, T], \mathbb{R})$ denotes the Banach space of all continuous functions defined on $[0, T] \times \mathbb{R}$ endowed with a topology of uniform convergence with the norm $\|x\|=$ $\sup _{t \in[0, T]}|x(t)|$.

By Lemma 3, we define an operator $\mathscr{P}: \mathscr{C} \rightarrow \mathscr{C}$ as

$$
\begin{aligned}
& (\mathscr{P} x)(t) \\
& =\frac{1}{\Gamma(q)} \int_{0}^{t}(t-s)^{q-1} f(s, x(s)) d s \\
& \quad-\frac{t^{n-1}}{\lambda \Gamma(q)} \int_{0}^{T}(T-s)^{q-1} f(s, x(s)) d s \\
& +\frac{t^{n-1}}{\lambda \Gamma(q)} \sum_{i=1}^{m} \frac{\gamma_{i}}{\Gamma\left(\beta_{i}\right)} \int_{0}^{\eta_{i}} \int_{0}^{s}\left(\eta_{i}-s\right)^{\beta_{i}-1}(s-u)^{q-1} \\
& \times f_{0}(u, x(u)) d u d s \\
& \quad-\int_{0}^{\zeta_{i}} \int_{i}^{s}\left(\zeta_{i}-s\right)^{\beta_{i}-1}(s-u)^{q-1} \\
& \times f(u, x(u)) d u d s],
\end{aligned}
$$

Observe that the problem (1) has a solution if and only if the associated fixed point problem $\mathscr{P} x=x$ has a fixed point.

In the first result we prove an existence and uniqueness result by means of Banach's contraction mapping principle. For the sake of convenience, we set

$$
\begin{aligned}
\Lambda= & \frac{T^{q}}{\Gamma(q+1)}+\frac{T^{q+n-1}}{|\lambda| \Gamma(q+1)} \\
& +\frac{T^{n-1}}{|\lambda|} \sum_{i=1}^{m} \gamma_{i} \frac{\eta_{i}^{q+\beta_{i}}-\zeta_{i}^{q+\beta_{i}}}{\Gamma\left(q+\beta_{i}+1\right)} .
\end{aligned}
$$

Theorem 4. Suppose that $f:[0, T] \times \mathbb{R} \rightarrow \mathbb{R}$ is a continuous function and satisfies the following assumption:

$\left(\mathrm{A}_{3}\right)$

$$
\begin{gathered}
|f(t, x)-f(t, y)| \leq L|x-y|, \\
\forall t \in[0,1], L>0, x, y \in \mathbb{R} .
\end{gathered}
$$

Then the boundary value problem (1) has a unique solution provided

$$
L<\frac{1}{\Lambda}
$$

where $\Lambda$ is given by (14). 
Proof. With $r \geq M \Lambda /(1-L \Lambda)$, we define $B_{r}=\{x \in \mathscr{C}$ : $\|x\| \leq r\}$, where $M=\sup _{t \in[0, T]}|f(t, 0)|<\infty$ and $\Lambda$ is given by (14). Then we show that $\mathscr{P} B_{r} \subset B_{r}$. For $x \in B_{r}$, by means of the inequality $|f(s, x(s))| \leq|f(s, x(s))-f(s, 0)|+|f(s, 0)| \leq$ $L\|x\|+M \leq L r+M$, it can easily be shown that

$$
\|\mathscr{P} x\|=(L r+M) \Lambda \leq r .
$$

Now, for $x, y \in \mathscr{C}$ and for each $t \in[0, T]$, we obtain

$$
\begin{aligned}
& \|(\mathscr{P} x)-(\mathscr{P} y)\| \\
& \leq \sup _{t \in[0, T]}\left\{\frac{1}{\Gamma(q)} \int_{0}^{t}(t-s)^{q-1}|f(s, x(s))-f(s, y(s))| d s\right. \\
& +\frac{t^{n-1}}{\lambda \Gamma(q)} \int_{0}^{T}(T-s)^{q-1} \\
& \times|f(s, x(s))-f(s, y(s))| d s \\
& +\frac{t^{n-1}}{\lambda \Gamma(q)} \\
& \times \sum_{i=1}^{m} \frac{\gamma_{i}}{\Gamma\left(\beta_{i}\right)} \\
& \times\left[\int_{0}^{\eta_{i}} \int_{0}^{s}\left(\eta_{i}-s\right)^{\beta_{i}-1}\right. \\
& \times(s-u)^{q-1} d u \\
& \times|f(u, x(u))-f(u, y(u))| d s \\
& -\int_{0}^{\zeta_{i}} \int_{0}^{s}\left(\zeta_{i}-s\right)^{\beta_{i}-1}(s-u)^{q-1} \\
& \times \mid f(u, x(u)) \\
& -f(u, y(u)) \mid d u d s]\} \\
& \leq L \Lambda\|x-y\| .
\end{aligned}
$$

Note that $\Lambda$ depends only on the parameters involved in the problem. As $L \Lambda<1$, therefore $\mathscr{P}$ is a contraction. Hence, by Banach's contraction mapping principle, the problem (1) has a unique solution on $[0, T]$.

Example 5. Let us consider the following 4-strip nonlocal boundary value problem:

$$
\begin{gathered}
{ }^{c} D^{9 / 2} x(t)=f(t, x(t)), \quad t \in[0,2], \\
x(0)=0, \quad x^{\prime}(0)=0, \quad x^{\prime \prime}(0)=0, \quad x^{\prime \prime \prime}(0)=0, \\
x(T)=\sum_{i=1}^{4} \gamma_{i}\left[I^{\beta i} x\left(\eta_{i}\right)-I^{\beta i} x\left(\zeta_{i}\right)\right],
\end{gathered}
$$

where $q=9 / 2, n=5, \zeta_{1}=1 / 4, \eta_{1}=1 / 2, \zeta_{2}=2 / 3, \eta_{2}=1$, $\zeta_{3}=5 / 4, \eta_{3}=4 / 3, \zeta_{4}=3 / 2, \eta_{4}=7 / 4, \gamma_{1}=5, \gamma_{2}=10$, $\gamma_{3}=15, \gamma_{4}=25, \beta_{1}=5 / 4, \beta_{2}=7 / 4, \beta_{3}=9 / 4, \beta_{4}=11 / 4$.

With the given values of the parameters involved, we find that

$$
\lambda=\left(T^{n-1}-\sum_{i=1}^{m} \gamma_{i} \frac{\left(\eta_{i}^{\beta_{i}+n-1}-\zeta_{i}^{\beta_{i}+n-1}\right) \Gamma(n)}{\Gamma\left(\beta_{i}+n\right)}\right)
$$

$\simeq 9.334784$

$$
\begin{aligned}
\Lambda & =\frac{T^{q}}{\Gamma(q+1)}+\frac{T^{q+n-1}}{|\lambda| \Gamma(q+1)}+\frac{T^{n-1}}{|\lambda|} \sum_{i=1}^{m} \gamma_{i} \frac{\eta_{i}^{q+\beta_{i}}-\zeta_{i}^{q+\beta_{i}}}{\Gamma\left(q+\beta_{i}+1\right)} \\
& \simeq 1.406972 .
\end{aligned}
$$

Let us choose

$$
f(t, x(t))=\frac{1}{\sqrt[3]{(t+8)}}\left(\tan ^{-1} x\right)+\sqrt{4+3 \sin 2 t}
$$

Clearly $L=1 / 2$ as $|f(t, x)-f(t, y)| \leq(1 / 2)|x-y|$ and $L<1 / \Lambda$, where $\Lambda \simeq 1.406972$. Therefore all the conditions of Theorem 4 hold and consequently there exists a unique solution for the problem (19) with $f(t, x(t))$ given by (21).

In case of the following unbounded nonlinear function:

$$
f(t, x(t))=\frac{x}{7}+\frac{1}{\sqrt[3]{(t+8)}}\left(\tan ^{-1} x\right)+\sqrt{4+3 \sin 2 t},
$$

we have $L=9 / 14$ and $L<1 / \Lambda(\Lambda \simeq 1.406972)$. As before, the problem (19) with $f(t, x(t))$ given by (22) has a unique solution.

In the second result we use the Leray-Schauder alternative.

Theorem 6 ((Leray-Schauder alternative) [33, page 4]). Let $X$ be a Banach space. Assume that $T: X \rightarrow X$ is completely continuous operator and the set

$$
V=\{u \in X \mid u=\mu T u, 0<\mu<1\}
$$

is bounded. Then $T$ has a fixed point in $X$.

Theorem 7. Assume that there exists a positive constant $L_{1}$ such that $|f(t, x)| \leq L_{1}$ for $t \in[0, T], x \in \mathbb{R}$. Then the problem (1) has at least one solution.

Proof. First of all, we show that the operator $\mathscr{P}$ is completely continuous. Note that the operator $\mathscr{P}$ is continuous in view 
of the continuity of $f$. Let $\mathscr{B} \subset \mathscr{C}$ be a bounded set. By the assumption that $|f(t, x)| \leq L_{1}$, for $x \in \mathscr{B}$, we have

$$
\begin{aligned}
& |(\mathscr{P} x)(t)| \\
& \leq \frac{1}{\Gamma(q)} \int_{0}^{t}(t-s)^{q-1}|f(s, x(s))| d s \\
& +\frac{t^{n-1}}{|\lambda| \Gamma(q)} \int_{0}^{T}(T-s)^{q-1}|f(s, x(s))| d s \\
& +\frac{t^{n-1}}{|\lambda| \Gamma(q)} \sum_{i=1}^{m} \frac{\gamma_{i}}{\Gamma\left(\beta_{i}\right)} \\
& \times\left[\int_{0}^{\eta_{i}} \int_{0}^{s}\left(\eta_{i}-s\right)^{\beta_{i}-1}(s-u)^{q-1}\right. \\
& \times|f(u, x(u))| d u d s \\
& -\int_{0}^{\zeta_{i}} \int_{0}^{s}\left(\zeta_{i}-s\right)^{\beta_{i}-1}(s-u)^{q-1} \\
& \times|f(u, x(u))| d u d s] \\
& \leq L_{1}\left[\frac{1}{\Gamma(q)} \int_{0}^{t}(t-s)^{q-1} d s+\frac{t^{n-1}}{|\lambda| \Gamma(q)} \int_{0}^{t}(T-s)^{q-1} d s\right. \\
& +\frac{t^{n-1}}{|\lambda| \Gamma(q)} \sum_{i=1}^{m} \frac{\gamma_{i}}{\Gamma\left(\beta_{i}\right)} \\
& \times\left[\int_{0}^{\eta_{i}} \int_{0}^{s}\left(\eta_{i}-s\right)^{\beta_{i}-1}\right. \\
& \times(s-u)^{q-1} d u d s \\
& -\int_{0}^{\zeta_{i}} \int_{0}^{s}\left(\zeta_{i}-s\right)^{\beta_{i}-1} \\
& \left.\left.\times(s-u)^{q-1} d u d s\right]\right] \\
& \leq L_{1}\left\{\frac{T^{q}}{\Gamma(q+1)}+\frac{T^{q+n-1}}{|\lambda| \Gamma(q+1)}\right. \\
& \left.+\frac{T^{n-1}}{|\lambda|} \sum_{i=1}^{m} \gamma_{i} \frac{\eta_{i}^{q+\beta_{i}}-\zeta_{i}^{q+\beta_{i}}}{\Gamma\left(q+\beta_{i}+1\right)}\right\}=L_{2},
\end{aligned}
$$

which implies that $\|(\mathscr{P} x)\| \leq L_{2}$. Further, we find that

$$
\begin{aligned}
& \left|(\mathscr{P} x)^{\prime}(t)\right| \\
& =\frac{1}{\Gamma(q-1)} \int_{0}^{t}(t-s)^{q-2}|f(s, x(s))| d s \\
& \quad+\frac{(n-1) t^{n-2}}{|\lambda| \Gamma(q)} \int_{0}^{T}(T-s)^{q-1}|f(s, x(s))| d s
\end{aligned}
$$

$$
\begin{aligned}
& +\frac{(n-1) t^{n-2}}{|\lambda| \Gamma(q)} \\
& \times \sum_{i=1}^{m} \frac{\gamma_{i}}{\Gamma\left(\beta_{i}\right)}\left[\int_{0}^{\eta_{i}} \int_{0}^{s}\left(\eta_{i}-s\right)^{\beta_{i}-1}(s-u)^{q-1}\right. \\
& \times|f(u, x(u))| d u d s \\
& -\int_{0}^{\zeta_{i}} \int_{0}^{s}\left(\zeta_{i}-s\right)^{\beta_{i}-1}(s-u)^{q-1} \\
& \times|f(u, x(u))| d u d s] \\
& \leq L_{1}\left[\frac{1}{\Gamma(q-1)} \int_{0}^{t}(t-s)^{q-2} d s\right. \\
& +\frac{(n-1) t^{n-2}}{|\lambda| \Gamma(q)} \int_{0}^{T}(T-s)^{q-1} d s \\
& +\frac{(n-1) t^{n-2}}{|\lambda| \Gamma(q)} \\
& \times \sum_{i=1}^{m} \frac{\gamma_{i}}{\Gamma\left(\beta_{i}\right)}\left(\int_{0}^{\eta_{i}} \int_{0}^{s}\left(\eta_{i}-s\right)^{\beta_{i}-1}\right. \\
& \times(s-u)^{q-1} \\
& \times|f(u, x(u))| d u d s \\
& -\int_{0}^{\zeta_{i}} \int_{0}^{s}\left(\zeta_{i}-s\right)^{\beta_{i}-1} \\
& \times(s-u)^{q-1} \\
& \times|f(u, x(u))| \\
& \times d u d s)] \\
& \leq L_{1}\left\{\frac{T^{q-1}}{\Gamma(q)}+\frac{(n-1) T^{q+n-2}}{|\lambda| \Gamma(q+1)}\right. \\
& \left.+\frac{(n-1) T^{n-2}}{|\lambda|} \sum_{i=1}^{m} \gamma_{j} \frac{\eta_{i}^{q+\beta_{i}}-\zeta_{i}^{q+\beta_{i}}}{\Gamma\left(q+\beta_{i}+1\right)}\right\}=L_{3} .
\end{aligned}
$$

Hence, for $t_{1}, t_{2} \in[0, T]$, we have

$$
\left|(\mathscr{P} x)\left(t_{2}\right)-(\mathscr{P} x)\left(t_{1}\right)\right| \leq \int_{t_{1}}^{t_{2}}\left|(\mathscr{P} x)^{\prime}(s)\right| d s \leq L_{3}\left(t_{2}-t_{1}\right) .
$$

This implies that $\mathscr{P}$ is equicontinuous on $[0, T]$. Thus, by the Arzelá-Ascoli theorem, the operator $\mathscr{P}: \mathscr{C} \rightarrow \mathscr{C}$ is completely continuous.

Next, we consider the set

$$
V=\left\{x \in \mathscr{C} \mid x=\mu \mathscr{P}_{x}, 0<\mu<1\right\},
$$


and show that the set $V$ is bounded. Let $x \in V$, then $x=$ $\mu \mathscr{P} x, 0<\mu<1$. For any $t \in[0, T]$, we have

$$
\begin{aligned}
& |x(t)|=\mu|(\mathscr{P} x)(t)| \\
& \leq \frac{1}{\Gamma(q)} \int_{0}^{t}(t-s)^{q-1}|f(s, x(s))| d s \\
& +\frac{t^{n-1}}{|\lambda| \Gamma(q)} \int_{0}^{T}(T-s)^{q-1}|f(s, x(s))| d s \\
& +\frac{t^{n-1}}{|\lambda| \Gamma(q)} \sum_{i=1}^{m} \frac{\gamma_{i}}{\Gamma\left(\beta_{i}\right)} \\
& \times\left[\int_{0}^{\eta_{i}} \int_{0}^{s}\left(\eta_{i}-s\right)^{\beta_{i}-1}(s-u)^{q-1}\right. \\
& \times|f(u, x(u))| d u d s \\
& -\int_{0}^{\zeta_{i}} \int_{0}^{s}\left(\zeta_{i}-s\right)^{\beta_{i}-1}(s-u)^{q-1} \\
& \times|f(u, x(u))| d u d s] \\
& \leq L_{1}\left\{\frac{T^{q}}{\Gamma(q+1)}+\frac{T^{q+n-1}}{|\lambda| \Gamma(q+1)}\right. \\
& \left.+\frac{T^{n-1}}{|\lambda|} \sum_{i=1}^{m} \gamma_{i} \frac{\eta_{i}^{q+\beta_{i}}-\zeta_{i}^{q+\beta_{i}}}{\Gamma\left(q+\beta_{i}+1\right)}\right\}=M_{1} .
\end{aligned}
$$

Thus, $\|x\| \leq M_{1}$ for any $t \in[0, T]$. So, the set $V$ is bounded. Thus, by the conclusion of Theorem 6 , the operator $\mathscr{P}$ has at least one fixed point, which implies that (1) has at least one solution.

Example 8. Consider the boundary value problem of Example 5 with

$$
f(t, x(t))=\frac{3 e^{\sqrt{(2-|x(t)|)^{3}}}\left[\cos 4 t+2 \ln \left(1+4 \sin ^{2} x(t)\right)\right]}{\sqrt{(10+\cos x(t))}} .
$$

Observe that $|f(t, x)| \leq L_{1}$ with $L_{1}=e^{2 \sqrt{2}}(1+\ln 25)$. Thus the hypothesis of Theorem 7 is satisfied. Hence by the conclusion of Theorem 7, the problem (19) with $f(t, x(t))$ given by (29) has at least one solution.

In the next we prove one more existence result for problem (1), based on the following known result.

Theorem 9 (see [34]). Let $X$ be a Banach space. Assume that $\Omega$ is an open bounded subset of $X$ with $\theta \in \Omega$ and let $T: \bar{\Omega} \rightarrow$ $X$ be a completely continuous operator such that

$$
\|T u\| \leq\|u\|, \quad \forall u \in \partial \Omega .
$$

Then $T$ has a fixed point in $\bar{\Omega}$.
Theorem 10. Let there exist a small positive number $\tau$ such that $|f(t, x)| \leq \nu|x|$ for $0<|x|<\tau$, with $0<\nu \leq 1 / \Lambda$, where $\Lambda$ is given by (14). Then the problem (1) has at least one solution.

Proof. Let us define $\mathscr{B}_{\tau}=\{x \in \mathscr{C} \mid\|x\|<\tau\}$ and take $x \in \mathscr{C}$ such that $\|x\|=\tau$, that is, $x \in \partial \mathscr{B}_{\tau}$. As before, it can be shown that $\mathscr{P}$ is completely continuous and

$$
\begin{aligned}
\|\mathscr{P} x\| \leq \sup _{t \in[0, t]}\{ & \frac{1}{\Gamma(q)} \int_{0}^{t}(t-s)^{q-1}|f(s, x(s))| d s \\
& +\frac{t^{n-1}}{|\lambda| \Gamma(q)} \\
& \times \int_{0}^{T}(T-s)^{q-1}|f(s, x(s))| d s \\
& +\frac{t^{n-1}}{|\lambda| \Gamma(q)} \\
& \times \sum_{i=1}^{m} \frac{\gamma_{i}}{\Gamma\left(\beta_{i}\right)} \\
& \times\left[\int_{0}^{\eta_{i}} \int_{0}^{s}\left(\eta_{i}-s\right)^{\beta_{i}-1}(s-u)^{q-1}\right. \\
& \quad \times|f(u, x(u))| d u d s \\
& \quad \int_{0}^{\zeta_{i}} \int_{0}^{s}\left(\zeta_{i}-s\right)^{\beta_{i}-1}(s-u)^{q-1}
\end{aligned}
$$

$\leq \Lambda v\|x\|$

which in view of the given condition $(\nu \Lambda \leq 1)$, gives $\|\mathscr{P} x\| \leq$ $\|x\|, x \in \partial \mathscr{B}_{\tau}$. Therefore, by Theorem 9 , the operator $\mathscr{P}$ has at least one fixed point, which in turn implies that the problem (1) has at least one solution.

Example 11. Consider the boundary value problem of Example 5 and let us consider

$$
\begin{aligned}
f(t, x(t))= & x\left(b^{5}+x^{4}(t)\right)^{1 / 5}+2\left(1+\cos \left(t^{4}+3\right)\right)^{5} \\
& \times(1-\cos x(t)), \quad x \neq 0, b>0 .
\end{aligned}
$$

For sufficiently small $x$ (ignoring $x^{2}$ and higher powers of $x$ ), we have

$$
\begin{aligned}
& \left|x\left(b^{5}+x^{4}(t)\right)^{1 / 5}+2\left(1+\cos \left(t^{4}+3\right)\right)^{5}(1-\cos x(t))\right| \\
& \quad \leq b|x| .
\end{aligned}
$$

Choosing $b \leq 1 / \Lambda$, all the assumptions of Theorem 10 hold. Therefore, the conclusion of Theorem 10 implies that the 
problem (19) with $f(t, x(t))$ given by (32) has at least one solution.

Our final existence result is based on Leray-Schauder nonlinear alternative.

Lemma 12 ((Nonlinear alternative for single valued maps) [33, page 135]). Let $E$ be a Banach space, $C$ a closed, convex subset of $E, U$ an open subset of $C$ and $0 \in U$. Suppose that $F: \bar{U} \rightarrow C$ is a continuous, compact (i.e., $F(\bar{U})$ is a relatively compact subset of C) map. Then either

(i) F has a fixed point in $\bar{U}$, or

(ii) there is a $u \in \partial U$ (the boundary of $U$ in $C$ ) and $\lambda \epsilon$ $(0,1)$ with $u=\lambda F(u)$.

Theorem 13. Assume that

$\left(\mathrm{A}_{1}\right)$ there exist a function $\sigma \in C\left([0,1], \mathbb{R}^{+}\right)$, and a nondecreasing function $\psi: \mathbb{R}^{+} \rightarrow \mathbb{R}^{+}$such that $|f(t, x)| \leq \sigma(t) \psi(\|x\|)$, for all $(t, x) \in[0, T] \times \mathbb{R} ;$

$\left(\mathrm{A}_{2}\right)$ there exists a constant $M>0$ such that

$$
\frac{M}{\psi(M) \Lambda\|\sigma\|}>1 \text {. }
$$

Then the boundary value problem (1) has at least one solution on $[0, T]$.

Proof. Consider the operator $\mathscr{P}: \mathscr{C} \rightarrow \mathscr{C}$ defined by (13). We show that $\mathscr{P}$ maps bounded sets into bounded sets in $C([0, T], \mathbb{R})$. For a positive number $r$, let $B_{r}=\{x \in$ $C([0, T], \mathbb{R}):\|x\| \leq r\}$ be a bounded set in $C([0, T], \mathbb{R})$. Then

$$
\begin{aligned}
&\|\mathscr{P} x\| \leq \sup _{t \in[0, T]}\{ \frac{1}{\Gamma(q)} \int_{0}^{t}(t-s)^{q-1}|f(s, x(s))| d s \\
&+\frac{t^{n-1}}{|\lambda| \Gamma(q)} \int_{0}^{T}(T-s)^{q-1}|f(s, x(s))| d s \\
&+\frac{t^{n-1}}{|\lambda| \Gamma(q)} \\
& \times \sum_{i=1}^{m} \frac{\gamma_{i}}{\Gamma\left(\beta_{i}\right)} \\
& \times\left[\int_{0}^{\eta_{i}} \int_{0}^{s}\left(\eta_{i}-s\right)^{\beta_{i}-1}\right. \\
& \times(s-u)^{q-1} \\
& \times|f(u, x(u))| d u d s \\
&-\int_{0}^{\zeta_{i}} \int_{0}^{s}\left(\zeta_{i}-s\right)^{\beta_{i}-1} \\
& \times(s-u)^{q-1}
\end{aligned}
$$

$$
\begin{aligned}
\leq \psi(r)\left\{\frac{T^{q}}{\Gamma(q+1)}+\frac{T^{q+n-1}}{|\lambda| \Gamma(q+1)}\right. \\
\left.+\frac{T^{n-1}}{|\lambda|} \sum_{i=1}^{m} \gamma_{i} \frac{\eta_{i}^{q+\beta_{i}}-\zeta_{i}^{q+\beta_{i}}}{\Gamma\left(q+\beta_{i}+1\right)}\right\}\|\sigma\| .
\end{aligned}
$$

Next we show that $F$ maps bounded sets into equicontinuous sets of $C([0,1], \mathbb{R})$. Let $t^{\prime}, t^{\prime \prime} \in[0,1]$ with $t^{\prime}<t^{\prime \prime}$ and $x \in B_{r}$, where $B_{r}$ is a bounded set of $C([0,1], \mathbb{R})$. Then we obtain

$$
\begin{aligned}
& \left|(\mathscr{P} x)\left(t^{\prime \prime}\right)-(\mathscr{P} x)\left(t^{\prime}\right)\right| \\
& =\mid \frac{1}{\Gamma(q)} \int_{0}^{t^{\prime \prime}}\left(t^{\prime \prime}-s\right)^{q-1} f(s, x(s)) d s \\
& -\frac{1}{\Gamma(q)} \int_{0}^{t^{\prime}}\left(t^{\prime}-s\right)^{q-1} f(s, x(s)) d s \\
& -\frac{\left[\left(t^{\prime \prime}\right)^{n-1}-\left(t^{\prime}\right)^{n-1}\right]}{\lambda \Gamma(q)} \int_{0}^{T}(T-s)^{q-1} f(s, x(s)) d s \\
& +\frac{\left[\left(t^{\prime \prime}\right)^{n-1}-\left(t^{\prime \prime}\right)^{n-1}\right]}{\lambda \Gamma(q)} \\
& \times \sum_{i=1}^{m} \frac{\gamma_{i}}{\Gamma\left(\beta_{i}\right)}\left[\int_{0}^{\eta_{i}} \int_{0}^{s}\left(\eta_{i}-s\right)^{\beta_{i}-1}(s-u)^{q-1}\right. \\
& \times|f(u, x(u))| d u d s \\
& -\int_{0}^{\zeta_{i}} \int_{0}^{s}\left(\zeta_{i}-s\right)^{\beta_{i}-1}(s-u)^{q-1} \\
& \times|f(u, x(u))| d u d s] \mid \\
& \leq \frac{1}{\Gamma(q)} \int_{0}^{t^{\prime}}\left|\left(t^{\prime \prime}-s\right)^{q-1}-\left(t^{\prime}-s\right)^{q-1}\right| \psi(r) \sigma(s) d s \\
& +\frac{1}{\Gamma(q)} \int_{t^{\prime}}^{t^{\prime \prime}}\left|t^{\prime \prime}-s\right|^{q-1} \psi(r) \sigma(s) d s \\
& +\frac{\left|\left(t^{\prime \prime}\right)^{n-1}-\left(t^{\prime}\right)^{n-1}\right|}{|\lambda| \Gamma(q)} \int_{0}^{T}|T-s|^{q-1} \psi(r) \sigma(s) d s \\
& +\frac{\left|\left(t^{\prime \prime}\right)^{n-1}-\left(t^{\prime}\right)^{n-1}\right|}{|\lambda| \Gamma(q)} \\
& \times \sum_{i=1}^{m} \frac{\gamma_{i}}{\Gamma\left(\beta_{i}\right)} \\
& \times\left[\int_{0}^{\eta_{i}} \int_{0}^{s}\left(\eta_{i}-s\right)^{\beta_{i}-1}(s-u)^{q-1} d u \psi(r) \sigma(s) d s\right. \\
& \left.-\int_{0}^{\zeta_{i}} \int_{0}^{s}\left(\zeta_{i}-s\right)^{\beta_{i}-1}(s-u)^{q-1} \psi(r) \sigma(s) d u d s\right]
\end{aligned}
$$


Obviously the right hand side of the above inequality tends to zero independently of $x \in B_{r}$ as $t^{\prime \prime}-t^{\prime} \rightarrow 0$. As $\mathscr{P}$ : $C([0, T], \mathbb{R}) \rightarrow C([0, T], \mathbb{R})$ satisfies the above assumptions, therefore it follows by the Arzelá-Ascoli theorem that $\mathscr{P}$ is completely continuous.

Let $x$ be a solution. Then, for $t \in[0, T]$, and following the similar computations as before, we find that

$$
\begin{aligned}
&|x(t)|=|\mu(\mathscr{P} x)(t)| \\
& \leq \psi(r)\left\{\frac{T^{q}}{\Gamma(q+1)}+\frac{T^{q+n-1}}{|\lambda| \Gamma(q+1)}\right. \\
&\left.+\frac{T^{n-1}}{|\lambda|} \sum_{i=1}^{m} \gamma_{i} \frac{\eta_{i}^{q+\beta_{i}}-\zeta_{i}^{q+\beta_{i}}}{\Gamma\left(q+\beta_{i}+1\right)}\right\}\|\sigma\| .
\end{aligned}
$$

In consequence, we have

$$
\frac{\|x\|}{\psi(\|x\|) \Lambda\|\sigma\|} \leq 1
$$

Thus, by $\left(\mathrm{A}_{2}\right)$, there exists $M$ such that $\|x\| \neq M$. Let us set

$$
V=\{x \in C([0, T], \mathbb{R}):\|x\|<M+1\} .
$$

Note that the operator $\mathscr{P}: \bar{V} \rightarrow C([0, T], \mathbb{R})$ is continuous and completely continuous. From the choice of $V$, there is no $x \in \partial V$ such that $x=\mu \mathscr{P}(x)$ for some $\mu \in(0,1)$. Consequently, by the nonlinear alternative of Leray-Schauder type (Lemma 12), we deduce that $\mathscr{P}$ has a fixed point $x \in \bar{V}$ which is a solution of the problem (1). This completes the proof.

Example 14. Consider the boundary value problem of Example 5 with

$$
f(t, x(t))=\frac{1}{\sqrt{t+4}}\left(1+\frac{|x|}{1+|x|}\right) \leq \sigma(t) \psi(\|x\|) .
$$

Then $\sigma(t)=1 / \sqrt{t+4}$ and $\psi(\|x\|)=2$. Using $\|\sigma\|=1 / 2, \Lambda \simeq$ 1.406972 , we find by the condition $\left(\mathrm{A}_{2}\right)$ that $M>\Lambda$. Thus all the assumptions of Theorem 13 are satisfied. Hence, it follows by Theorem 13 that the problem (19) with $f(t, x(t))$ defined by (40) has at least one solution.

If we choose an unbounded nonlinearity as follows:

$$
f(t, x(t))=\frac{1}{\sqrt{t+4}}\left(1+\frac{|x|}{1+|x|}+\frac{|x|}{2}\right) .
$$

Then $f(t, x(t)) \leq \sigma(t) \psi(\|x\|)$ with $\sigma(t)=1 / \sqrt{t+4}$ and $\psi(\|x\|)=2+\|x\| / 2$. Using the earlier arguments, with $\|\sigma\|=$ $1 / 2, \Lambda \simeq 1.406972$, we find that $M>M_{1}, M_{1} \approx 2.170392$. Hence the problem (19) with $f(t, x(t))$ given by (41) has at least one solution.

\section{Acknowledgment}

The authors are grateful to the anonymous referees for their valuable comments.

\section{References}

[1] K. S. Miller and B. Ross, An Introduction to the Fractional Calculus and Differential Equations, A Wiley-Interscience Publication, John Wiley \& Sons, New York, NY, USA, 1993.

[2] S. G. Samko, A. A. Kilbas, and O. I. Marichev, Fractional Integrals and Derivatives, Gordon and Breach Science Publishers, Yverdon, Switzerland, 1993.

[3] I. Podlubny, Fractional Differential Equations, vol. 198 of Mathematics in Science and Engineering, Academic Press, San Diego, Calif, USA, 1999.

[4] A. A. Kilbas, H. M. Srivastava, and J. J. Trujillo, Theory and Applications of Fractional Differential Equations, vol. 204 of North-Holland Mathematics Studies, Elsevier Science B.V., Amsterdam, The Netherlands, 2006.

[5] V. Lakshmikantham, S. Leela, and J. V. Devi, Theory of Fractional Dynamic Systems, Cambridge Academic, Cambridge, UK, 2009.

[6] D. Baleanu, K. Diethelm, E. Scalas, and J. J. Trujillo, Fractional Calculus Models and Numerical Methods, vol. 3 of Series on Complexity, Nonlinearity and Chaos, World Scientific, Boston, Mass, USA, 2012.

[7] M. Benchohra, S. Hamani, and S. K. Ntouyas, "Boundary value problems for differential equations with fractional order and nonlocal conditions," Nonlinear Analysis: Theory, Methods \& Applications, vol. 71, no. 7-8, pp. 2391-2396, 2009.

[8] R. P. Agarwal, M. Benchohra, and S. Hamani, "A survey on existence results for boundary value problems of nonlinear fractional differential equations and inclusions," Acta Applicandae Mathematicae, vol. 109, no. 3, pp. 973-1033, 2010.

[9] K. Balachandran and J. J. Trujillo, "The nonlocal Cauchy problem for nonlinear fractional integrodifferential equations in Banach spaces," Nonlinear Analysis: Theory, Methods \& Applications, vol. 72, no. 12, pp. 4587-4593, 2010.

[10] D. Băleanu and O. G. Mustafa, "On the global existence of solutions to a class of fractional differential equations," Computers \& Mathematics with Applications, vol. 59, no. 5, pp. 1835-1841, 2010.

[11] W. Zhong and W. Lin, "Nonlocal and multiple-point boundary value problem for fractional differential equations," Computers \& Mathematics with Applications, vol. 59, no. 3, pp. 1345-1351, 2010.

[12] V. Gafiychuk and B. Datsko, "Mathematical modeling of different types of instabilities in time fractional reaction-diffusion systems," Computers \& Mathematics with Applications, vol. 59, no. 3, pp. 1101-1107, 2010.

[13] B. Ahmad and J. J. Nieto, "Riemann-Liouville fractional integrodifferential equations with fractional nonlocal integral boundary conditions," Boundary Value Problems, vol. 2011, article 36, 9 pages, 2011.

[14] B. Ahmad and S. K. Ntouyas, "A four-point nonlocal integral boundary value problem for fractional differential equations of arbitrary order," Electronic Journal of Qualitative Theory of Differential Equations, vol. 2011, no. 22, 15 pages, 2011.

[15] C. Cuevas, H. Soto, and A. Sepúlveda, "Almost periodic and pseudo-almost periodic solutions to fractional differential and integro-differential equations," Applied Mathematics and Computation, vol. 218, no. 5, pp. 1735-1745, 2011.

[16] N. J. Ford and M. L. Morgado, "Fractional boundary value problems: analysis and numerical methods," Fractional Calculus and Applied Analysis, vol. 14, no. 4, pp. 554-567, 2011.

[17] B. Ahmad and S. K. Ntouyas, "A note on fractional differential equations with fractional separated boundary conditions," 
Abstract and Applied Analysis, vol. 2012, Article ID 818703, 11 pages, 2012.

[18] A. Aghajani, Y. Jalilian, and J. J. Trujillo, "On the existence of solutions of fractional integro-differential equations," Fractional Calculus and Applied Analysis, vol. 15, no. 1, pp. 44-69, 2012.

[19] W. Sudsutad and J. Tariboon, "Existence results of fractional integro-differential equations with $\mathrm{m}$-point multi-term fractional order integral boundary conditions," Boundary Value Problems, vol. 2012, article 94, 2012.

[20] B. Ahmad and S. K. Ntouyas, "Existence results for nonlocal boundary value problems of fractional differential equations and inclusions with strip conditions," Boundary Value Problems, vol. 2012, article 55, 2012.

[21] B. Ahmad and J. J. Nieto, "Riemann-liouville fractional differential equations with fractional boundary conditions," Fixed Point Theory, vol. 13, pp. 329-336, 2012.

[22] B. Ahmad, J. J. Nieto, A. Alsaedi, and M. El-Shahed, "A study of nonlinear Langevin equation involving two fractional orders in different intervals," Nonlinear Analysis: Real World Applications, vol. 13, no. 2, pp. 599-606, 2012.

[23] A. Cabada and G. Wang, "Positive solutions of nonlinear fractional differential equations with integral boundary value conditions," Journal of Mathematical Analysis and Applications, vol. 389, no. 1, pp. 403-411, 2012.

[24] B. Ahmad, T. Hayat, and S. Asghar, "Diffraction of a plane wave by an elastic knife-edge adjacent to a rigid strip," The Canadian Applied Mathematics Quarterly, vol. 9, no. 4, pp. 303-316, 2001.

[25] S. Asghar, B. Ahmad, and M. Ayub, "Diffraction from an absorbing half plane due to a finite cylindrical source," AcusticaActa Acustica, vol. 82, pp. 365-367, 1996.

[26] G. S. Wang and A. F. Blom, "A strip model for fatigue crack growth predictions under general load conditions," Engineering Fracture Mechanics, vol. 40, no. 3, pp. 507-533, 1991.

[27] H. Jiang, F. Liu, I. Turner, and K. Burrage, "Analytical solutions for the multi-term time-space Caputo-Riesz fractional advection-diffusion equations on a finite domain," Journal of Mathematical Analysis and Applications, vol. 389, no. 2, pp. 1117-1127, 2012.

[28] E. Yusufoglu and I. Turhan, "A mixed boundary value problem in orthotropic strip containing a crack," Journal of the Franklin Institute, vol. 349, no. 9, pp. 2750-2769, 2012.

[29] Dh. Chang and A. Kotousov, "A strip yield model for two collinear cracks," Engineering Fracture Mechanics, vol. 90, pp. 121-128, 2012.

[30] B. Lebental and F. Bourquin, "Visco-acoustic modelling of a vibrating plate interacting with water confined in a domain of micrometric size," Journal of Sound and Vibration, vol. 331, no. 8, pp. 1870-1886, 2012.

[31] T. V. Renterghem, D. Botteldooren, and K. Verheyen, "Road traffic noise shielding by vegetation belts of limited depth," Journal of Sound and Vibration, vol. 331, no. 10, pp. 2404-2425, 2012.

[32] J. Poblet-Puig and A. Rodrguez-Ferran, "Modal-based prediction of sound transmission through slits and openings between rooms," Journal of Sound and Vibration, vol. 332, pp. 1265-1287, 2012.

[33] A. Granas and J. Dugundji, Fixed Point Theory, Springer Monographs in Mathematics, Springer, New York, NY, USA, 2005.

[34] D. R. Smart, Fixed Point Theorems, Cambridge University Press, Cambridge, UK, 1980. 


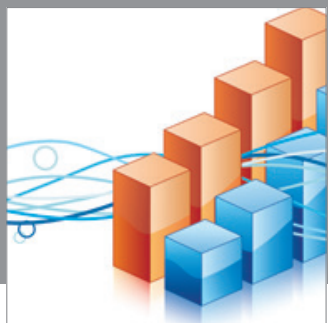

Advances in

Operations Research

mansans

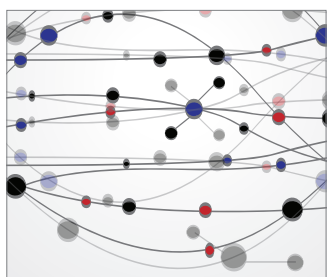

The Scientific World Journal
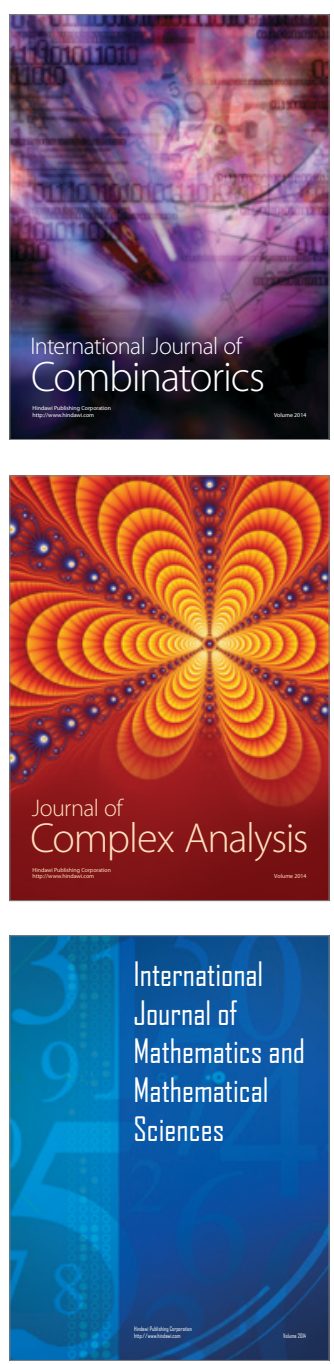
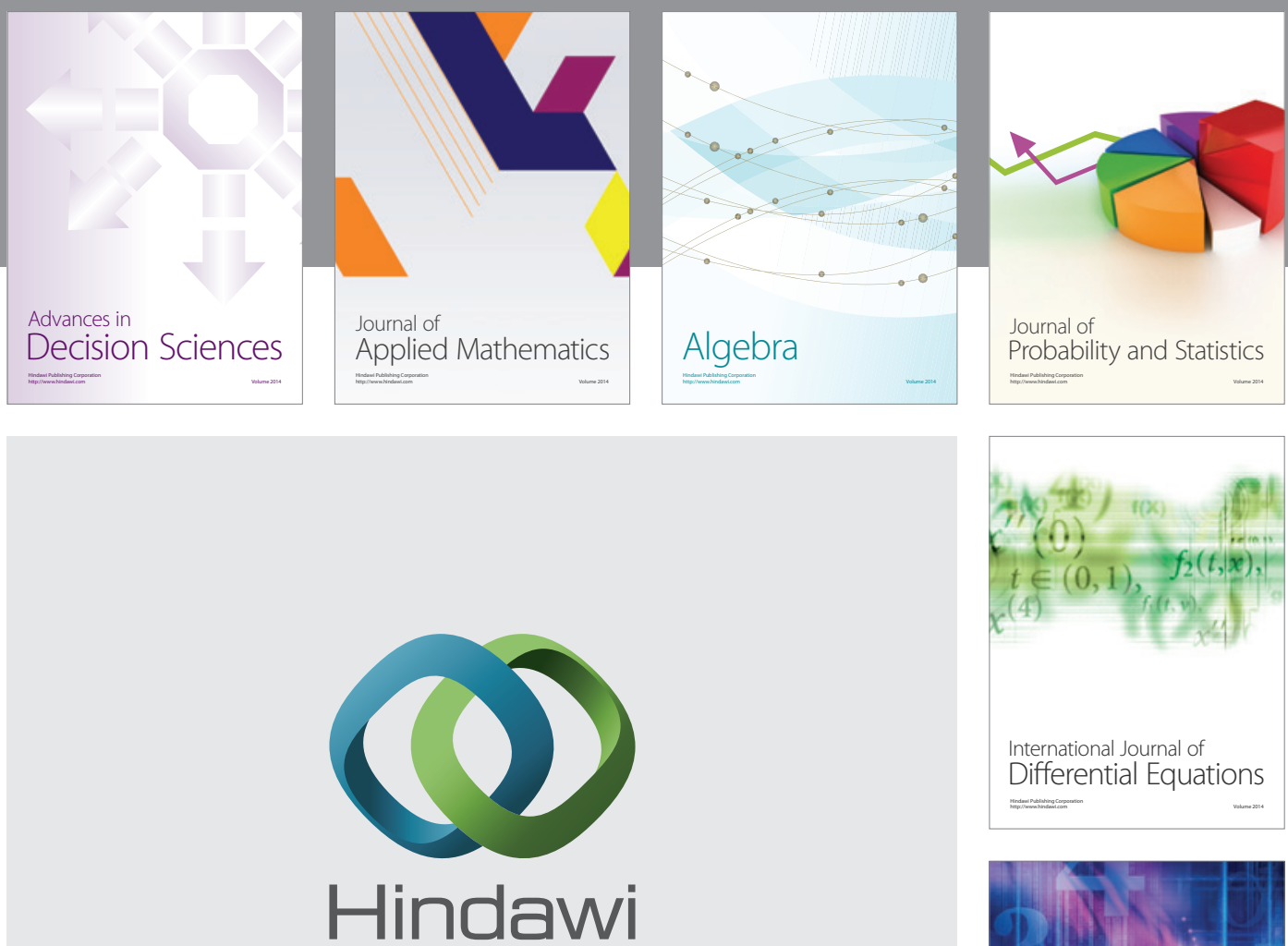

Submit your manuscripts at http://www.hindawi.com
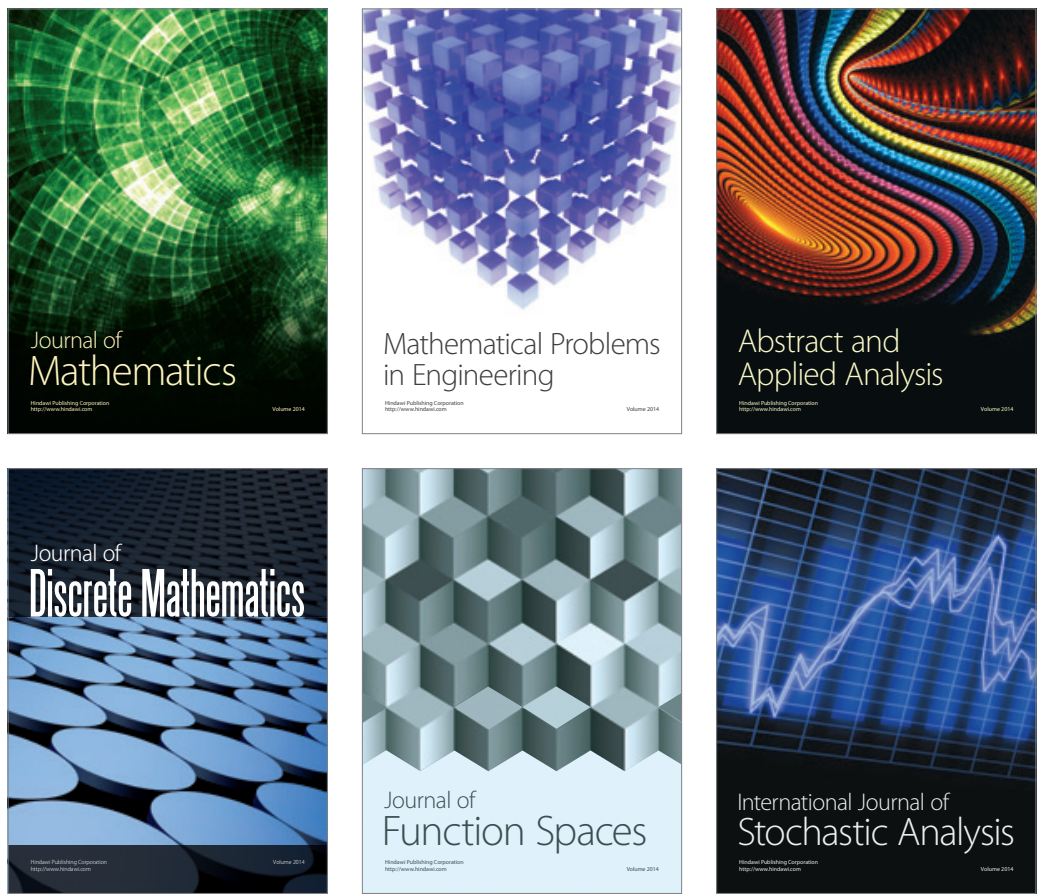

Journal of

Function Spaces

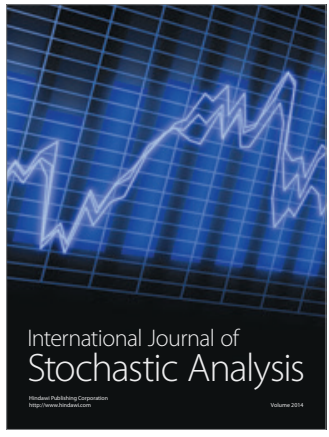

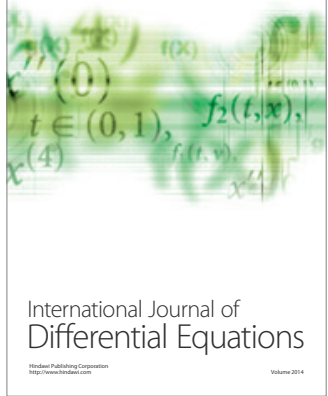
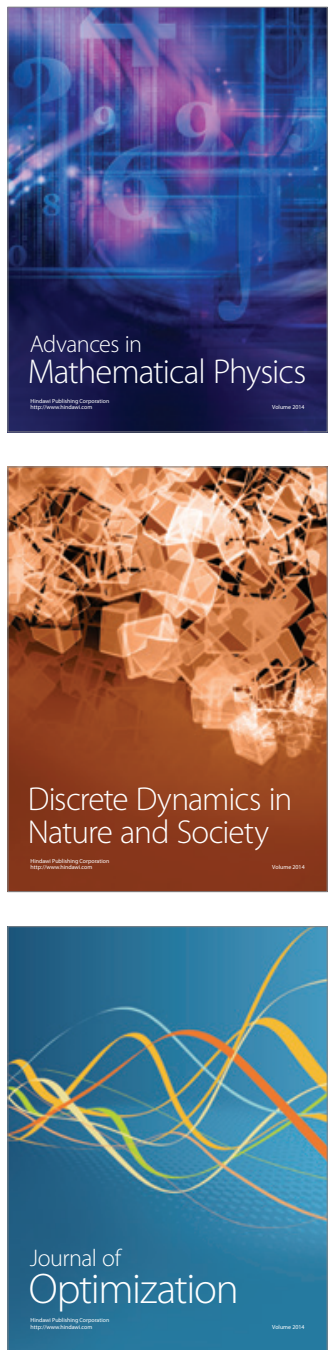